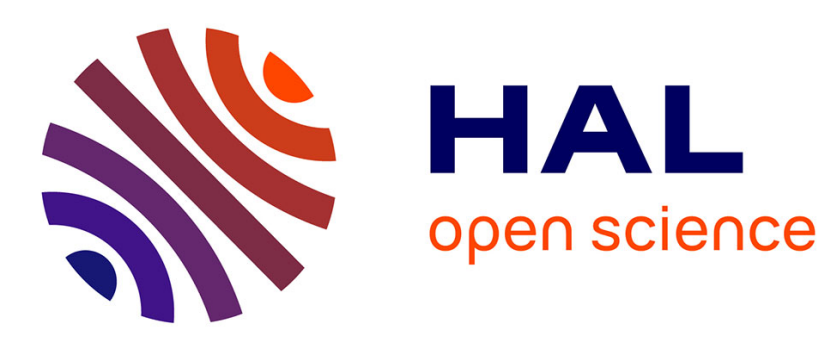

\title{
Managing temporal allocation in Integrated Modular Avionics
}

Nesrine Badache, Katia Jaffres-Runser, Jean-Luc Scharbarg, Christian Fraboul

\section{To cite this version:}

Nesrine Badache, Katia Jaffres-Runser, Jean-Luc Scharbarg, Christian Fraboul. Managing temporal allocation in Integrated Modular Avionics. 19th IEEE International Conference on Emerging Technologies and Factory Automation (ETFA 2014), Sep 2014, Barcelone, Spain. pp. 1-8, 10.1109/ETFA.2014.7005225 . hal-01359995

\section{HAL Id: hal-01359995 https://hal.science/hal-01359995}

Submitted on 5 Sep 2016

HAL is a multi-disciplinary open access archive for the deposit and dissemination of scientific research documents, whether they are published or not. The documents may come from teaching and research institutions in France or abroad, or from public or private research centers.
L'archive ouverte pluridisciplinaire HAL, est destinée au dépôt et à la diffusion de documents scientifiques de niveau recherche, publiés ou non, émanant des établissements d'enseignement et de recherche français ou étrangers, des laboratoires publics ou privés. 


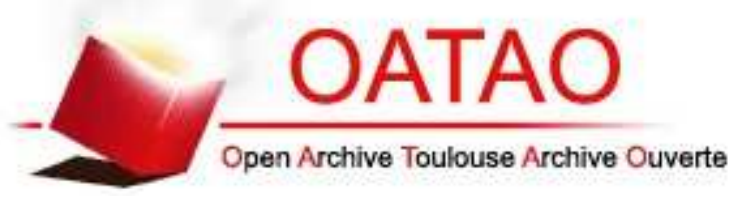

\section{Open Archive TOULOUSE Archive Ouverte (OATAO)}

OATAO is an open access repository that collects the work of Toulouse researchers and makes it freely available over the web where possible.

This is an author-deposited version published in : http://oatao.univ-toulouse.fr/ Eprints ID : 15187

The contribution was presented at ETFA 2014:

http://eeyem.eap.gr/en/content/19th-ieee-international-conference-emergingtechnologies-and-factory-automation-etfa-2014

To cite this version : Badache, Nesrine and Jaffres-Runser, Katia and Scharbarg, Jean-Luc and Fraboul, Christian Managing temporal allocation in Integrated Modular Avionics. (2014) In: 19th IEEE Internationa 1 Conference on Emerging Technologies and Factory Automation (ETFA 2014), 16 September 2014 - 19 September 2014 (Barcelone, Spain).

Any correspondence concerning this service should be sent to the repository administrator: staff-oatao@listes-diff.inp-toulouse.fr 


\title{
Managing temporal allocation in Integrated Modular Avionics
}

\author{
Nesrine Badache, Katia Jaffrès-Runser, Jean-Luc Scharbarg and Christian Fraboul \\ University of Toulouse \\ IRIT-INPT/ENSEEIHT \\ 2, rue Charles Camichel, 31000 Toulouse, France \\ \{nesrine.badache, katia.jaffresrunser, jean-luc.scharbarg, christian.fraboul\}@enseeiht.fr
}

\begin{abstract}
Recent civil airborne platforms are produced using Integrated Modular Avionics (IMA). IMA promotes both sharing of execution and communication resources by the avionics applications. Designs following IMA decrease the weight of avionics equipment and improve the whole system scalability. However, the price to pay for these benefits is an increase of the system's complexity, triggering a challenging system integration process. Central to this integration step are the timing requirements of avionics applications: the system integrator has to find a mapping of applications and communications on the available target architecture (processing modules, networks, etc.) such as end-to-end delay constraints are met. These challenges stress the need for a tool capable of evaluating different integration choices in the early design stages of IMA.

In this paper, we present and formalize the problem of spatial and temporal integration of an IMA system. Then, we focus on the temporal allocation problem which is critical to ensure a proper timely behavior of the system. Two main properties are presented to ensure perfect data transmission for hard real-time flows. To quantify the quality of a set of valid temporal allocations, CPM utilization and communication robustness performance criteria are defined. We show on an example that both criteria are antagonist and that they can be leveraged to choose an allocation that either improves the system computing performance or the robustness of the network.
\end{abstract}

\section{INTRODUCTION}

Embedded avionics systems have evolved from a federative architecture where calculators dedicated to avionics applications were interconnected through dedicated mono-emitter links towards a modular and distributed architecture.

An IMA architecture interconnects several spatially distributed processing units, sensors and actuators using one or more communication networks. Processing units communicate most of the time using an AFDX network (Avionic Full Duplex Switched Ethernet) which has been standardized in ARINC 664 [3]. Avionics applications are distributed over the set of processing units called core processing modules. Current integration choices are made thanks to the experience and know-how of specialists. These specialists have limited tools to guide such a complex and crucial design process. However, such systems encompass around a hundred CPMs, exchanging a thousand of flows. This magnitude clearly calls for a guided design and integration process.

In the literature, only few works have proposed solutions to the IMA integration problem. Lauer et al. [6] have modeled the IMA architecture using formal methods and calculated worst case network traversal times using trajectory approach to verify the timing requirements of the whole system. This approach has scalability issues as it relies on formal verification. Al Sheikh et al. [9] propose a mixed integer linear program to optimize the spatial and temporal integration choices with resource constraints. Solutions obtained with this approach are unfortunately not robust to the asynchronism of the modules. More specifically, the calculated solution holds for a set of module startup offset, but may not work anymore for a different set of offsets. Moreover, the approach does not scale up to the size of future larger IMA architectures.

The work presented herein is a step ahead of our previous studies presented in [10] and [7]. This paper focuses on the derivation of the execution period of partitions which are receiving time-constrained data from distant source partitions. The proposed approach completely mitigates message losses for all flows in the network by introducing two constraints on the end-to-end communication delay. We show that several allocations meet these constraints and that it is not always obvious to decide which solution is the most interesting one for the system integrator.

The paper is organized as follows. Section II introduces the IMA integration issues while Section III relies on a worst case analysis to define the constraints that ensure failure-free message deliveries. Section IV illustrates these constraints on a practical example and exhibits the need for a more precise performance evaluation. Next, Section V introduces CPM utilization and communication robustness criteria. Finally, Section VI concludes this paper.

\section{IMA INTEGRATION ISSUES}

\section{A. Integrated Modular Avionics (IMA)}

The Integrated Modular Avionics (IMA) architecture has been developed in the late 2000s for civil avionics systems. It has been standardized as ARINC 651 [1] for the definition of the generic hardware architecture and as ARINC 653 [2] for the corresponding software architecture. The design goal of the software architecture, called APEX (APplication EXecutive interface), is to enable spatial and temporal partitioning of the avionics functions for the target architecture. 
IMA is a distributed architecture where processing units, called Core Processing Modules (or CPM) are interconnected through embedded communication networks. An avionics application is composed of a set of functions or tasks which may be executed on a single or on several CPMs, distributively. In this latter case, two tasks executed on two distant CPMs communicate through the so-called APEX communication ports to create an APEX logical channel. These APEX ports are reminiscent of standard IP world sockets. Logical ports are mapped to a physical interface of the underlying communication network.

To ensure physical and temporal segregation of memory and computing resources, a static resource allocation of the avionics tasks is enabled by APEX. APEX defines an execution time window that repeats periodically on a CPM to execute a set of tasks grouped in partitions. A partition $P_{i}$ is characterized by its execution duration $b_{i}$ and its period $T_{i}$. A set of tasks is assigned by the integrator to a partition. Consequently, this set of tasks is executed with the periodicity of the hosting partition and their total execution duration is bounded by the duration of the hosting partition as well. The set of all partitions hosted on the same CPM are scheduled in a cyclical frame called MAjor time Frame (MAF). The length of a MAF is given by the least common multiple of the partition periods. Several instances of the same partition may be executed within a MAF.

Each partition is assigned a dedicated and reserved memory and computing space used for both data storage and execution. Thus, the tasks assigned to two different partitions can not access the same memory space

Two distant communicating tasks belong to two distinct communicating partitions. A communication is unidirectional, with one source partition and one destination partition. Data are emitted periodically, following the source partition execution period.

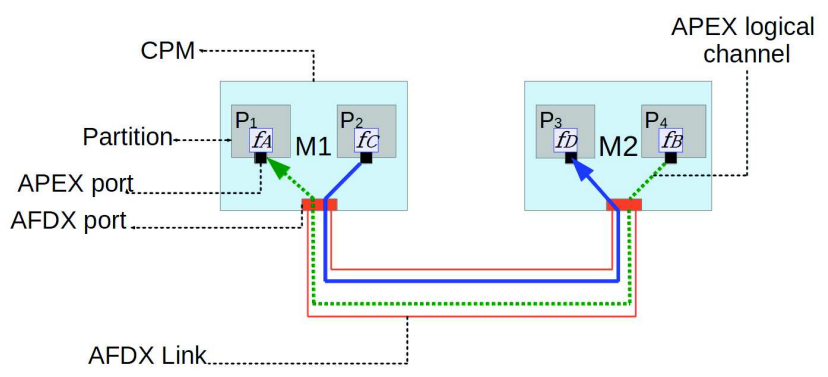

Fig. 1. IMA integration example

Figure 1 pictures a toy example of an IMA system composed of two CPMs, hosting two partitions each. In each partition, only one task is being executed. Tasks $f_{B}$ and $f_{A}$ (resp. $f_{C}$ and $f_{D}$ ) belong to the same avionics application. $f_{B}$ emits data to $f_{A}$ using its emission APEX port, which is mapped to an AFDX virtual link (the dotted green line) connected to the receiving partition $P_{1}$, hosting function $f_{A}$. Similarly, $f_{C}$ emits to $f_{D}$ using another AFDX virtual link (in solid blue). Figure 2 represents a possible MAF of the first CPM hosting the two partitions $P_{1}$ and $P_{2}$. As illustrated, partition $P_{1}$ repeats with period $T_{1}$

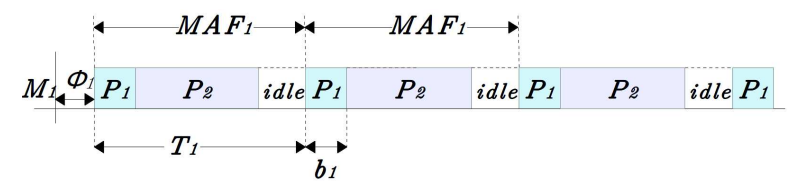

Fig. 2. MAF illustration

\section{B. IMA design and integration issues}

Given a target architecture described by the layout of CPMs and networks, and a set of applications (e.g., flight warning, automatic cruise control, etc.), the integration process has to solve several problems. The first problems are related to the physical allocation of the applications to the available CPMs and networks. It decomposes into two main steps:

The spatial allocation of applications to CPMs. An avionics application is first segmented into a set of partitions hosting its processing functions. Each partition has to be assigned to a CPM knowing that each CPM has an available memory space budget and a given processing speed.

The spatial allocation of APEX logical channels to networking resources. If an application is deployed over several CPMs, their functions communicate via the network using APEX channels. The APEX logical channels defined between the distant applications have to be mapped to the available communication channels provided by the embedded networks of the target architecture (i.e. AFDX virtual links, ARINC 429 links, etc.).

Once a possible spatial allocation of the applications is set, the integrator knows on which CPMs each partition of each application runs. He knows as well which worst case networking latencies are expected between two communicating partitions [12], [11]. The data emitted by each source partition is timely constrained by a freshness parameter $(F P)$. Once the data leaves the output APEX port of a partition, a counter is armed. The data has to be consumed by its destination partition before this freshness duration has elapsed. This constraint has to be ensured for each set of communicating partition. To meet these constraints and accept the possible spatial allocation under study, a valid temporal allocation of the destination partitions periods has to be found, which is discussed next.

Temporal period allocation of destination partitions. Since the applications set the period of the source partitions, only the period of destination partitions can be tuned by the system integrator to ensure proper message delivery between the two communicating partitions. The destination period can be adjusted to compensate for possible jitters introduced by the network. Moreover, as explained above, allocated partitions on each CPM are grouped into a MAF, which is given by the least common multiple of the partition periods. Thus, by allocating periods of destination partitions, the MAF is modified as well.

This paper concentrates on the temporal allocation of partitions. These temporal integration issues are complex to solve 
for current avionics systems which are typically composed of more than a hundred of CPMs, interconnected by thousands of virtual links. To tackle this problem, we introduce first two specific constraints that ensure, knowing the worst case communication delay, that no data is lost for a given partitionto-partition communication. Then, we illustrate on an example that several temporal allocations meet these constraints. To characterize the efficiency of these admissible solutions, we introduce two performance criteria that measure the delay margin of the flows and the MAF occupancy percentage. The intuition behind these criteria is to be able to select solutions which are more robust to a possible future increase in network jitters or to select solutions which provide room for adding new partitions in the CPMs. Proposed criteria could be combined in the future to extract solutions that trade-off both metrics, using a multi-objective optimization heuristic.

\section{TEMPORAL PERIOD INTEGRATION: WORST CASE ANALYSIS}

This section defines first the IMA system model we consider, and then recalls the precise definition of the end-to-end functional delay of [6]. This delay is the partition-to-partition communication delay which is constrained by the freshness parameter introduced previously. Next, two constraints on the destination period are introduced that ensure perfect message receptions for a partition to partition communication. We prove that if a temporal period allocation fulfills these two constraints, no message can be lost.

\section{A. IMA system model}

An avionics application is represented in the following as a set $\mathcal{P}=\left\{P_{1}, \ldots, P_{n}\right\}$ of partitions that have to be allocated and scheduled on a set $\mathcal{M}=\left\{M_{1}, \ldots, M_{m}\right\}$ of parallel CPMs. Each partition $P_{i} \in \mathcal{P}$ is characterized by the number of functions it hosts. In this work, we assume that one partition hosts one and only one function. Formally, partition $P_{i}$ is defined by its execution period $T_{i}$ and duration $b_{i}$. We define with $d_{n}$ the date of the $n^{\text {th }}$ partition activation.

In its partition, we assume a function $f$ first reads the messages coming from one or more source functions (source functions may be distant or local), then processes its data and at the end of $b_{i}$, sends its messages on the APEX port. Function $f$ is characterized by its worst case execution time. Since we assume that a partition only hosts one function, its worst case execution time is equal to the duration $b_{i}$ of the partition $P_{i}$ it belongs to.

IMA system is completely distributed: CPMs have different start-up dates. These different start-up dates are modeled by relative offsets. The offset of CPM $M_{\ell}$ is denoted by $\phi_{\ell}$ (cf. Fig. 2).

The communication between a source partition $P_{i}$ and a destination partition $P_{j}$ is defined as an APEX logical channels denoted $\mathrm{Com}_{i, j}$. Com $i, j$ is constrained by a freshness parameter $F P_{i, j}$. We recall that it is the maximum time a message can wait before being consumed at the destination function from its emission date at the source APEX port.

\section{B. End-to-end functional communication delay}

We consider a communication $\operatorname{Com}_{i, j}$ represented in Figure 3 between a sender partition $P_{i}$ allocated on CPM $M_{\ell}$, and a destination partition $P_{j}$ allocated on CPM $M_{k}$. The source partition $P_{i}$ emits periodically, at the end of its execution duration, an occurrence of the message flow $\mathrm{msg}$ that must be read at the next activation of $P_{j}$. The $n^{t h}$ occurrence of the flow $m s g$ is denoted by $m s g_{n}$.

The end-to-end functional communication delay [6] is the sum of the following latencies as illustrated in Fig. 3:

- Source buffering LATEncy : A message occurrence $m s g_{n}$ generated by a sensor connected to the module $M_{\ell}$ may have to wait for the source partition $P_{i}$ to be active before being consumed in the buffer.

- Source execution duration : Partition $P_{i}$ is active for $b_{i}$ units of time. During this source execution duration, $m s g_{n}$ stays at the source partition until it is sent to the APEX output port.

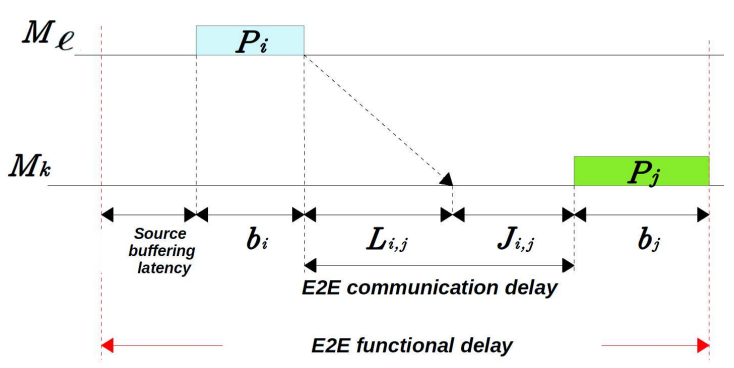

Fig. 3. End-to-end functional and communication delays

- Network LATEncy : Each occurrence $m s g_{n}$ experiences a network latency denoted by $L_{i, j}$ on its communication channel $\operatorname{Com}_{i, j}$. We assume here that this latency $L_{i, j}$ belongs to the interval $\left[L_{\min }, L_{\max }\right]$, where $L_{\min }$ is the best case network latency and $L_{\max }$ is the worst case network latency on $\mathrm{Com}_{i, j}$. The network latency on each APEX logical channel is bounded which is compatible with well-know worst case calculation studies done in the context of avionics embedded networking [11], [12]. Notice that $L_{\min }$ and $L_{\max }$ depend as well on indices $i$ and $j$ but for clarity purposes, they have been omitted in this paper.

- Destination Latency : Once $m s g_{n}$ arrives at the destination CPM, it may have to wait for the partition $P_{j}$ to become active and being read by the destination function. The duration between the date $m s g_{n}$ arrives at $M_{k}$ and the date it is consumed by $P_{j}$ is defined as the destination latency. The occurrences $m s g_{n}$ that are received at the destination partition do not constitute a periodic flow anymore. This is a consequence of the variable network latency and the difference in start-up dates of CPMs.

- Destination execution duration : Once $m s g_{n}$ is received by $P_{j}$, it is processed for a duration of $b_{j}$ units of time. 
We define herein as well the end-to-end communication delay (denoted as $E 2 E_{i, j}$ ) which is the sum of the network and destination latencies as shown on Fig. 3. This latency is the one which has to be lower or equal than the freshness parameter $F P_{i, j}$ for $m s g_{n}$ to be considered as valid. The end-to-end communication delay is central for the derivations proposed in the rest of this paper.

\section{Worst case destination period analysis}

Considering these different latencies and variabilities in the end-to-end communication delay, two constraints on the destination partition periods are introduced that ensure a timely and failure free message delivery.

A message occurrence $m s g_{n}$ can be lost for two reasons:

- $m s g_{n}$ experiences an end-to-end communication delay larger than $F P_{i, j}$ (cf. Fig. 4-(a)),

- $m s g_{n}$ is overwritten by $m s g_{n+1}$ before being read by $P_{j}$ (cf. Fig. 4-(b))

Occurrence $m s g_{n}$ is overwritten by $m s g_{n+1}$ if the two following conditions occur: i) $\mathrm{msg}_{n}$ arrives too late to be consumed by the activation of $P_{j}$ immediately following the activation of $P_{i}$ (in this case, it has to wait an extra period $T_{j}$ for the next activation of $P_{j}$ ) and $i i$ ) if during this extra waiting time, a newer occurrence $m s g_{n+1}$ arrives at $P_{j}$ and overwrites $m s g_{n}$ in the destination buffer.

To sum-up, message losses may occur if the resulting end-to-end communication delay is greater than the required freshness parameter $F P_{i, j}$ ), or if an other occurrence $m s g_{n+1}$ is received before the consumption of $m s g_{n}$.

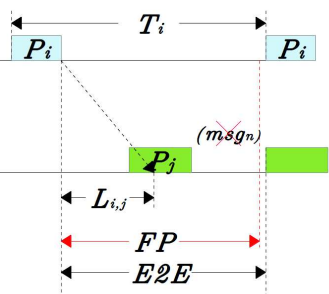

(a)

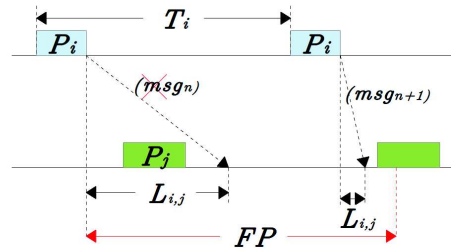

(b)
Fig. 4. $P_{i}$ and $P_{j}$ communication

To avoid such losses of messages, the destination execution period $T_{j}$ of $P_{j}$ has to be adjusted so that these two loss cases never happen. We show next that these requirements are met if following Properties 1 and 2 hold:

Property 1. A message does not violate its freshness parameter $F P_{i, j}$ if and only if:

$$
T_{j} \leq F P_{i, j}-L_{\max }
$$

Proof:

In order to meet the freshness constraint $F P_{i, j}$ associated to $C_{C o m}, j$, the end-to-end communication delay of the message $m s g_{n}$, denoted $E 2 E_{n}$ must not exceed $F P_{i, j}$ :

$$
E 2 E_{n} \leq F P_{i, j}
$$

By definition, we have:

$$
L_{i, j}+J_{i, j} \leq F P_{i, j}
$$

In the worst case, $L_{i, j}$ is equal to $L_{\max }$ and $m s g_{n}$ has to wait an entire period $T_{j}$ (e.g. $m s g_{n}$ arrives just after the destination partition has been activated). Thus, $J_{i, j}$ is equal to $T_{j}$ in the worst case and:

$$
L_{\max }+T_{j} \leq F P_{i, j}
$$

From Eq. (2), it can be deduced that if the destination period exceeds $F P_{i, j}-L_{\max }$, the freshness constraint is not met for $m s g_{n}$. In other words, valid values of $T_{j}$ have to follow:

$$
T_{j} \leq F P_{i, j}-L_{\max }
$$

If partition $P_{j}$ is the destination partition of $K$ different source partitions, its period $T_{j}$ has to be adjusted considering the most restrictive freshness parameter among the $K$ required ones. In this case, Property 1 holds by replacing $F P_{i, j}$ with $\min _{k}\left(F P_{k, j}\right)$.

Property 2. A message $\mathrm{msg}_{n}$ is never overwritten by a newer occurrence $\mathrm{msg}_{n+1}$ if and only if

$$
T_{j}<T_{i}-\left(L_{\max }-L_{\min }\right)
$$

The destination period $T_{j}$ has to be reduced to prevent the reception of two occurrences of $m s g$ between two successive executions of $P_{j}$.

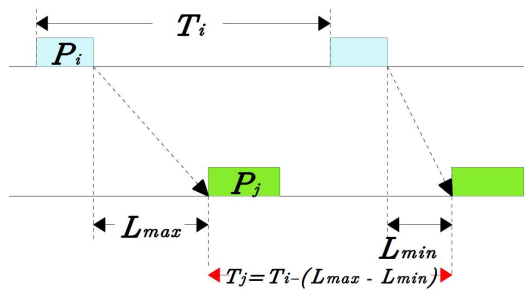

Fig. 5. Minimal execution period

Proof:

Two successive occurrences of $m s g, m s g_{n}$ and $m s g_{n+1}$, are sent by $P_{i}$.

Occurrences $m s g_{n}$ and $m s g_{n+1}$ should respectively be received at the destination partition $P_{j}$ at the dates $d_{n}$ (the $n^{\text {th }}$ activation of $\left.P_{i}\right)$ and $d_{n+1}$ (the $(n+1)^{t h}$ activation of $\left.P_{i}\right)$. These dates are defined by:

$$
\begin{gathered}
d_{n}=\phi_{\ell}+n T_{i}+b_{i}+L_{n} \\
d_{n+1}=\phi_{\ell}+(n+1) T_{i}+b_{i}+L_{n+1}
\end{gathered}
$$

where $L_{n}$ and $L_{n+1}$ are the network latencies experienced by $m s g_{n}$ and $m s g_{n+1}$, respectively.

The destination partition $P_{j}$ reads both occurrences of $m s g$ if the destination period $T_{j}$ follows:

$$
T_{j} \leqslant\left(d_{n+1}-d_{n}\right)
$$


After substituting $d_{n+1}$ and $d_{n}$ with their definition, we have:

$$
T_{j} \leqslant T_{i}+L_{n+1}-L_{n}
$$

The destination period has to mitigate messages losses for the must constraining case. This case happens for the smallest possible value of $d_{n+1}-d_{n}$, i.e. the smallest possible value of $T_{i}+L_{n+1}-L_{n}$. This smallest value is experienced if $L_{n}=$ $L_{\max }$ and $L_{n+1}=L_{\min }$. Thus:

$$
\begin{gathered}
T_{j} \leqslant T_{i}+L_{\text {min }}-L_{\text {max }} \\
\Leftrightarrow T_{j} \leqslant T_{i}-\left(L_{\text {max }}-L_{\text {min }}\right)
\end{gathered}
$$

If partition $P_{j}$ is the destination partition of $K$ different source partitions, the most constraining communication is the one with the smallest period $T_{i}$. Thus, Property 2 holds in this case by replacing $T_{i}$ with $\min _{k}\left(T_{k}\right)$.

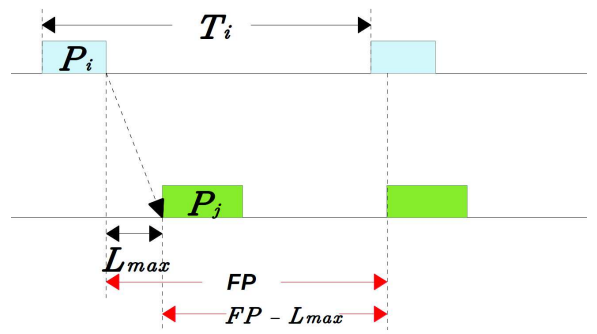

Fig. 6. Oversampling of $P_{j}$ period

The two properties are combined by setting a maximum possible destination period equal to the minimum of the constraints imposed by Property 1 and 2. In other words,

$$
T_{j} \leqslant T_{j}^{\max }
$$

with $T_{j}^{\max }=\min \left(F P_{i, j}-L_{\max }, T_{i}-\left(L_{\max }-L_{\min }\right)\right)$.

To conclude, any value of $T_{j}$ smaller than $T P_{j}^{\max }$ is a valid setting where no message occurrences can be lost. As we will show in the next example, if small values of $T_{j}$ that meet Eq. (4) are chosen, some activations of $P_{j}$ may never receive any message occurrence. Thus, destination period adjustment comes at the price of overusing the CPM at the destination as already noticed in [8] for the FlexRay network static segment configuration. Another drawback is that there is as well less room for adding other partitions to the destination MAF. However, this oversampling has a beneficial effect: if the network jitter increases (i.e. $L_{\max }$ increases) because of some evolution in the network load, the current temporal allocation is still robust enough to mitigate occurrence losses due to this increase of $L_{\max }$. Both effects will be illustrated on an example in the next Section.

\section{IMA TEMPORAL ALLOCATION EXAMPLE}

The temporal allocation problem is illustrated on the example of Fig. 7. It represents the spatial allocation of an application on four CPMs $M_{1}, \ldots, M_{4}$ interconnected by an AFDX network. We consider that this application is part of

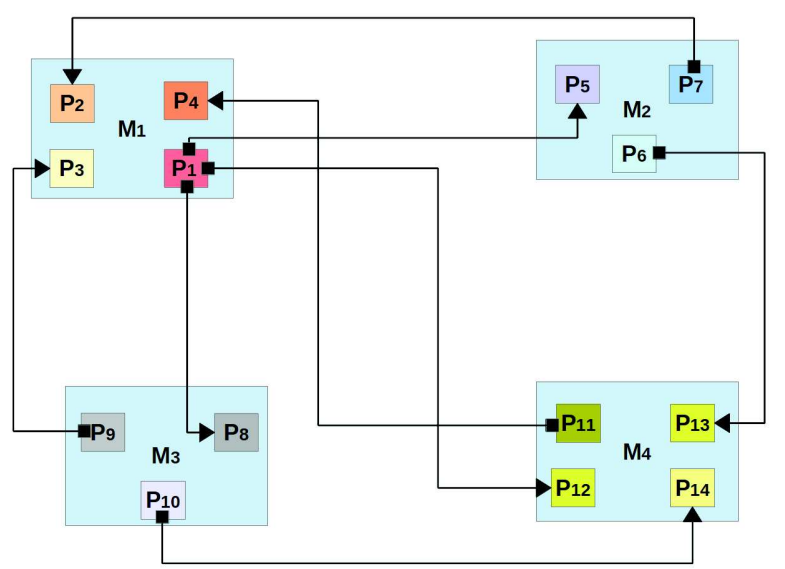

Fig. 7. Example of IMA system

a much larger IMA system which includes other modules hosting other avionics applications.

The application under study decomposes into 14 partitions. As shown in Fig. 7, there are 6 source partitions: $P_{1}$ executes on CPM $M_{1}, P_{6}$ and $P_{7}$ on $M_{2}, P_{9}$ and $P_{10}$ on $M_{3}, P_{11}$ on $M_{4}$. Execution periods of these source partitions are imposed by the application. They are listed in Table I.

\begin{tabular}{|l|c|c|c|}
\cline { 2 - 4 } \multicolumn{1}{c|}{} & Periods $T_{i}(\mathrm{~ms})$ & BAG $(\mathrm{ms})$ & Destination \\
\hline$P_{1}$ & 120 & 64 & $P_{5}, P_{8}, P_{12}$ \\
$P_{6}$ & 60 & 32 & $P_{13}$ \\
$P_{7}$ & 60 & 32 & $P_{2}$ \\
$P_{9}$ & 60 & 32 & $P_{3}$ \\
$P_{10}$ & 60 & 32 & $P_{14}$ \\
$P_{11}$ & 40 & 32 & $P_{4}$ \\
\hline
\end{tabular}

TABLE I

SOURCE PARTITION FEATURES

As stated previously, a source partition generates one message occurrence at the end of each of its execution. These occurrences are transmitted to at least one destination partition via an AFDX virtual link. An AFDX virtual link is characterized by its Bandwidth Allocation Gap (BAG) which is the minimum authorized duration between two consecutive message transmissions on the link. In Table I, the BAG of the virtual links are set to be no greater than the period of their corresponding source partitions. Thus, it follows that a message occurrence can never be delayed because of the BAG of its corresponding virtual link. Table I lists as well the destination partitions of each source partition.

The execution duration of all the partitions are given in Table II. Each partition can be activated more than one time in the MAF of its CPM.

The temporal destination period allocation of this configuration has to meet the constraints presented in Section III which are conditioned by the network latency variability $\left(L_{\min }\right.$ and $\left.L_{\max }\right)$ and the freshness parameter $F P$. These values for our example are given in Table III. 


\begin{tabular}{|l|c|}
\cline { 2 - 2 } \multicolumn{1}{c|}{} & Execution durations $b_{i}(\mathrm{~ms})$ \\
\hline$P_{1}$ & 5 \\
$P_{2}$ & 10 \\
$P_{3}$ & 10 \\
$P_{4}$ & 5 \\
$P_{5}$ & 15 \\
$P_{6}$ & 15 \\
$P_{7}$ & 15 \\
$P_{8}$ & 15 \\
$P_{9}$ & 15 \\
$P_{10}$ & 15 \\
$P_{11}$ & 10 \\
$P_{12}$ & 10 \\
$P_{13}$ & 10 \\
$P_{14}$ & 10 \\
\hline
\end{tabular}

TABLE II

EXECUTION DURATIONS OF THE PARTITIONS

\begin{tabular}{|l|c|c|c|}
\cline { 2 - 4 } \multicolumn{1}{c|}{} & $L_{\min }(\mathrm{ms})$ & $L_{\max }(\mathrm{ms})$ & Freshness $F P_{i, j}(\mathrm{~ms})$ \\
\hline $\operatorname{Com}_{1,5}$ & 2 & 12 & 100 \\
$\operatorname{Com}_{1,8}$ & 3 & 15 & 100 \\
$\operatorname{Com}_{1,12}$ & 1 & 6 & 100 \\
$\operatorname{Com}_{6,13}$ & 1 & 6 & 60 \\
$\operatorname{Com}_{7,2}$ & 2 & 12 & 60 \\
$\operatorname{Com}_{9,3}$ & 4 & 20 & 60 \\
$\operatorname{Com}_{10,14}$ & 2 & 10 & 60 \\
$\operatorname{Com}_{11,4}$ & 1 & 5 & 40 \\
\hline
\end{tabular}

TABLE III

LATENCIES AND FRESHNESS CONSTRAINTS

Thanks to Properties 1 and 2, the maximum allowed destination period $T_{j}^{\max }$ has to be calculated for each destination partition following Eq. (4). Let's detail this computation on partition $P_{5}$ whose source partition is $P_{1}$. From Property 1, we have:

$$
\begin{aligned}
T_{5} & <F P_{1}-L_{1}^{\max } \\
& <100-12 \\
& <88 \mathrm{~ms}
\end{aligned}
$$

From Property 2, we have:

$$
\begin{aligned}
T_{5} & <T_{1}-\left(L_{1}^{\max }-L_{1}^{\min }\right) \\
& <120-(12-2) \\
& <110 \mathrm{~ms}
\end{aligned}
$$

Thus, the period of $P_{5}$ must be equal to at most $T_{5}^{\max }=$ $88 \mathrm{~ms}$. Results for other destination partitions are given in Table IV which lists the maximum allowed period to meet Property 1, 2 and both at the same time.

The following step consists in building the MAFs associated to each CPM by choosing the periods of the destination partitions. The destination partitions periods have of course to be lower than $T_{j}^{\max }$ but to simplify the scheduling inside the MAF and keep MAFs to a limited size, partition periods are chosen to be harmonic as well. For each CPM, a solution is given by the set of destination partition periods that respect these constraints (we recall source partition periods are set and can not be changed). Several solutions may fulfill these constraints of course.

\begin{tabular}{|l|c|c|c|}
\cline { 2 - 4 } \multicolumn{1}{c|}{} & $T_{j}^{\text {max }}$ & Property 1 & Property 2 \\
\hline$P_{2}$ & 48 & 48 & 50 \\
$P_{3}$ & 40 & 40 & 44 \\
$P_{4}$ & 35 & 35 & 36 \\
$P_{5}$ & 88 & 88 & 110 \\
$P_{8}$ & 85 & 85 & 108 \\
$P_{12}$ & 94 & 94 & 115 \\
$P_{13}$ & 54 & 54 & 55 \\
$P_{14}$ & 50 & 50 & 52 \\
\hline
\end{tabular}

TABLE IV

DESTINATION PARTITION CONSTRAINTS

In our example, following solutions are chosen per CPM:

- For module $M_{2}$, source partitions $P_{6}$ and $P_{7}$ have a period of $60 \mathrm{~ms}$. We recall that destination partition period $T_{5}$ must not exceed $88 \mathrm{~ms}$. Thus, one valid solution is to assign a period of $60 \mathrm{~ms}$ to the three partitions.

- The same assignment can be done for partitions $P_{8}, P_{9}$ and $P_{10}$ on module $M_{3}$,

- For module $M_{4}$, a similar reasoning leads to a period of $40 \mathrm{~ms}$ for partitions $P_{11}, P_{13}$ and $P_{14}$, and to a period of $80 \mathrm{~ms}$ for $P_{12}$. The larger period of 80 is chosen for $P_{12}$ because $T_{12}^{\max }=94 \mathrm{~ms}$ and 80 is the largest allowed value which is harmonic with $T_{11}=40 \mathrm{~ms}$. Choosing a lower period for $P_{12}$ is possible, but it clearly wastes too much processing time at $M_{4}$.

- The case of module $M_{1}$ is a bit more complex. The period of source partition $P_{1}$ is set to $120 \mathrm{~ms}$ by the application, while periods of $P_{2}, P_{3}$ and $P_{4}$ can not exceed $48 \mathrm{~ms}$, $40 \mathrm{~ms}$ and $35 \mathrm{~ms}$, respectively. Thus, two solutions are possible:

1) $P_{2}$ and $P_{3}$ are assigned a period of $40 \mathrm{~ms}$ and $P_{4}$ a period of $20 \mathrm{~ms}$,

2) $P_{2}, P_{3}$ and $P_{4}$ are assigned a period of $30 \mathrm{~ms}$.

Overall, it leads to two different period assignments on $M_{1}$ (which are not the only ones possible). Figure 8 shows that both assignments lead to schedulable partitions on CPMs by representing the MAFs obtained for each CPM. The main question raised by this example is how to choose between both valid allocations of CPM $M_{1}$. This question is discussed next.

\section{Performance EVAluation CRITERIA FOR TEMPORAL ALLOCATION}

\section{A. Analysis of the valid allocations for $M_{1}$}

Looking more closely at the MAFs of Fig. 8, it can be seen that the MAF obtained with the first allocation proposed for $M_{1}$ (i.e. where $\left(T_{2}, T_{3}, T_{4}\right)=(40,40,20)$ ) has more idle space than the one of the second allocation (i.e. where $\left.\left(T_{2}, T_{3}, T_{4}\right)=(30,30,30)\right)$. For both solutions, the same number of message occurrences have to be absorbed by the partitions hosted on $M_{1}$, thus, allocation 1 uses the computation power more efficiently than allocation 2 .

Another analysis perspective is to look at the networking performance. There are three communications ending at $M_{1}$ : 


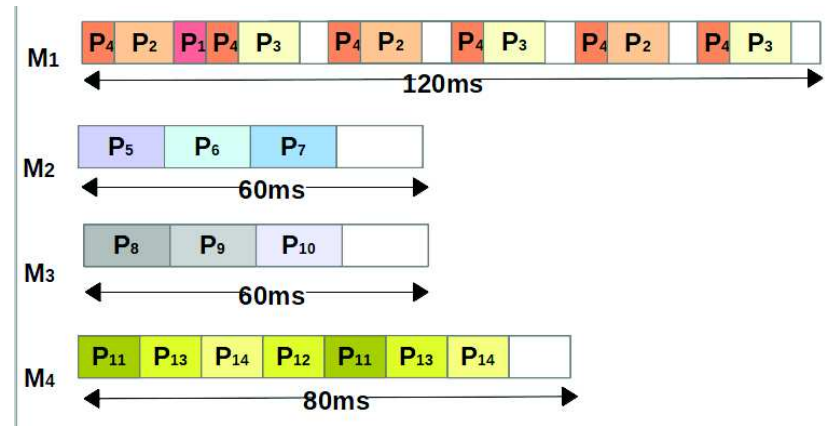

(a)

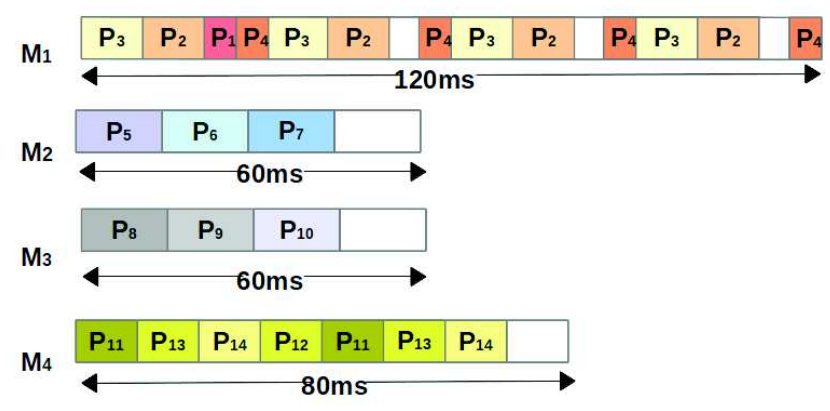

(b)

Fig. 8. Two possible integrations in $M_{1}$

$\mathrm{Com}_{7,2}, \mathrm{Com}_{9,3}$ and $\mathrm{Com}_{11,4}$. For each communications, it is possible to calculate theirs worst case end-to-end communication delay $\left(E 2 E_{w c}\right)$ which is the sum of their $L_{\max }$ value and the destination period chosen either with allocation 1 or allocation 2 .

\begin{tabular}{|l|c|c|c|}
\cline { 2 - 4 } \multicolumn{1}{c|}{} & $E 2 E_{w c}(\mathrm{~ms})$ & Freshness $F P_{i, j}(\mathrm{~ms})$ & Delay margin \\
\hline $\operatorname{Com}_{7,2}$ & 52 & 60 & 8 \\
$\operatorname{Com}_{9,3}$ & 60 & 60 & 0 \\
$\operatorname{Com}_{1,4}$ & 25 & 40 & 15 \\
\hline
\end{tabular}

TABLE V

WORST CASE E2E DELAY, FRESHNESS PARAMETER AND DELAY MARGIN FOR COMMUNICATIONS ENDING IN $M_{1}$ WITH $\left(T_{2}, T_{3}, T_{4}\right)=(40,40,20)$.

For instance, for $\mathrm{Com}_{7,2}$ using allocation 1, we have $L_{\max }=12 \mathrm{~ms}$ and $T_{2}=40 \mathrm{~ms}$. Thus, $E 2 E_{w c}=52 \mathrm{~ms}$. Still for $\mathrm{Com}_{7,2}$, using allocation 2 this time, $T_{2}=30 \mathrm{~ms}$ and $E 2 E_{w c}=42 \mathrm{~ms}$. Compared to the required freshness parameter, allocation 2 provides a larger end-to-end communication delay margin than allocation 1 for $\mathrm{Com}_{7,2}$. The same analysis has been done for the other communications ending at $M_{1}$ and results can be found in Tables V and VI, for allocations 1 and 2 respectively.

\begin{tabular}{|l|c|c|c|}
\cline { 2 - 4 } \multicolumn{1}{c|}{} & $E 2 E_{w c}(\mathrm{~ms})$ & Freshness $F P_{i, j}(\mathrm{~ms})$ & Delay margin \\
\hline $\operatorname{Com}_{7,2}$ & 42 & 60 & 18 \\
$\operatorname{Com}_{9,3}$ & 50 & 60 & 10 \\
$\operatorname{Com}_{11,4}$ & 35 & 40 & 5 \\
\hline
\end{tabular}

TABLE VI

WORST CASE E2E DELAY, FRESHNESS PARAMETER AND DELAY MARGIN FOR COMMUNICATIONS ENDING IN $M_{1}$ WITH $\left(T_{2}, T_{3}, T_{4}\right)=(30,30,30)$.

From Tables V and VI, it can be seen that the delay margin for allocation 2 is larger in average than for allocation 1. Moreover, the smallest delay margin of allocation 1 is of 0 $\mathrm{ms}$ while the smallest one for allocation 2 is of $5 \mathrm{~ms}$. Clearly, allocation 2 provides a better networking performance than allocation 1. If for some reason the network configuration has to be modified, and if this introduces an increase of $L_{\max }$ of at most $5 \mathrm{~ms}$ per VL, we can conclude that allocation 2 is still valid and no changes in the MAFs have to be made.

Thus, even though allocation 1 has a more efficient use of the CPM computation power, it is less robust from the networking performance point of view. Both allocations represent two different trade-off between network robustness and CPM utilization. To capture these two features, we propose next to introduce two performance criteria that can be leveraged for a future IMA system-wide multi-objective optimization.

\section{B. CPM utilization factor and communication robustness}

This Section defines two performance evaluation criteria capturing the two previously introduces features for a given temporal period allocation:

- $C P M$ utilization factor $Q_{i}$ : For a CPM $M_{i}$, the utilization factor is defined as the percentage of time the CPM is executing a partition. For instance, in Fig. $8, M_{2}$ and $M_{3}$ have a utilization factor of 0.75 each. Formally:

$$
Q_{i}=\frac{\sum_{P_{k} \in M_{i}} b_{k}}{b_{M A F}}
$$

where $b_{M A F}$ is the MAF duration.

- Communication robustness $\delta_{i, j}$ : For each communication, we calculate the margin between the worst case end-toend communication delay and its corresponding freshness parameter. If this margin is very small, any change in the network configuration may lead to losses and the whole system may have to be re-designed. Formally:

$$
\delta_{i, j}=F P_{i, j}-E 2 E_{w c}=F P_{i, j}-\left(L_{\max }+T_{j}\right)
$$

\section{System-wide metrics}

The previously defined elementary metrics measure either the performance of an allocation at the CPM level or at the communication level. It is useful to define a metric to characterize the global performance of the whole IMA system under study. Several options are possible and we introduce two of them.

In the first option, it possible to define a global metric by averaging the elementary metrics. Global average metrics are formally defined by: 
Global average CPM utilization factor $Q^{a v g}$ :

$$
Q^{a v g}=\frac{\sum_{i \in[1 . . m]} Q_{i}}{m}
$$

with $m$ is the number of CPMs in the system.

Global average communication robustness $\Delta^{a v g}$ :

$$
\Delta^{a v g}=\frac{\sum_{(i, j)} \delta_{i, j}}{n_{c o m}}
$$

where $n_{\text {com }}$ is the number of communications in the system.

Another option is to select the worst elementary metric as representative of the system performance. In this case, the global metrics are given by:

Global worst CPM utilization factor $Q^{w}$ :

$$
Q^{w}=\max _{i \in[1 . . m]} Q_{i}
$$

Global worst communication robustness $\Delta^{w}$ :

$$
\Delta^{w}=\min _{(i, j)} \delta_{i, j}
$$

For the communication robustness, it is in our opinion more interesting to use the global $\Delta^{w}$ metric compared to the average one because it clearly identifies the communication that may be detrimental to the overall performance. On the contrary, for the CPM utilisation, we are more interested in the average load of the system and the peak load.

It is possible to illustrate the global metrics $Q^{a v g}$ and $\Delta^{w}$ for allocations 1 and 2 on the previous example. For allocation $1, Q^{a v g}=0,79$ while for allocation $2, Q^{\text {avg }}=0,81$. The system-wide CPM utilization criteria clearly shows that allocation 1 is more efficient than allocation 2 .

Looking at the communication robustness, $\Delta^{w}=0 \mathrm{~ms}$ for the first allocation. This very small global margin is caused by $\operatorname{Com}_{9,3}$. For allocation 2, $\Delta^{w}=5 \mathrm{~ms}$. It is communication $\mathrm{Com}_{11,4}$ that experiences a margin of 5. This criteria clearly shows that allocation 2 has a better communication robustness, which is in line with our previous analysis. The performance of both allocations is illustrated in the 2-dimensional space of $\left(Q^{a v g}, \Delta^{w}\right)$ in Fig. 9. The best trade-off is obtained for low values of $Q^{\text {avg }}$ and high values of $\Delta^{w}$.

To conclude, we have illustrated these metrics on a smallscale example. In practice, more than thousands of flows and hundreds of CPMs are embedded into an avionics IMA platform. Thus, managing the temporal allocation is a very large and complex problem. There is a clear need for an automated help for the design of such systems. In this paper, we have analyzed the temporal allocation problem and provided system-wide performance analysis criteria. These criteria can now be leveraged to optimize the temporal allocation problem. Since both CPM utilization and communication robustness criteria are antagonist by nature, multi-objective optimization can be addressed to provide different trade-off solutions to the system integrator.

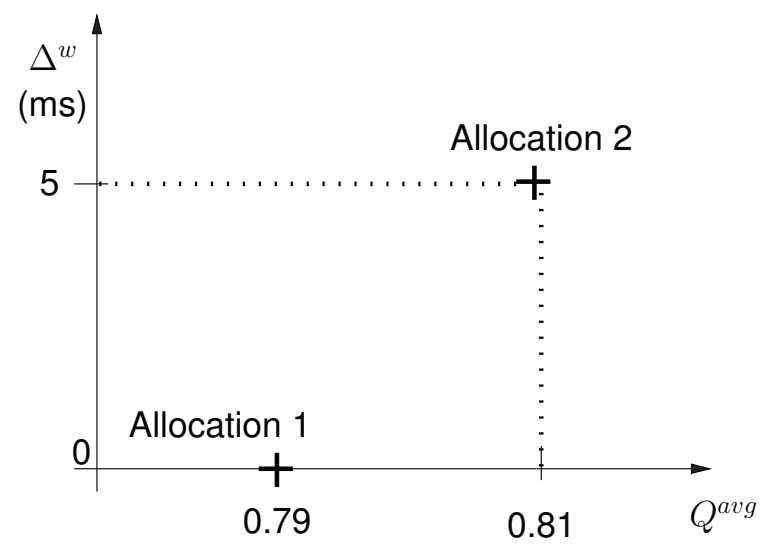

Fig. 9. Multi-objective performance of allocation 1 and 2 for $\left(Q^{a v g}, \Delta^{w}\right)$.

\section{CONCLUSION}

In this paper, the issues related to the spatial and temporal integration of an IMA system are introduced. For the temporal period allocation problem, two main properties have been proved that ensure perfect data transmission for all timeconstrained flows of the system. Next, we have proposed two criteria, naming CPM utilization and communication robustness, to quantify the quality of valid temporal allocations. All these contributions have been illustrated on a practical example. Future works will concentrate on defining and solving a multi-objective optimization problem leveraging the performance criteria presented in this paper.

\section{REFERENCES}

[1] Aeronautical Radio Inc. ARINC 651, Design Guidance for Integrated Modular Avionics, 1991.

[2] Aeronautical Radio Inc. ARINC 653, Avionics Application Software Standard Interface, 1997.

[3] Aeronautical Radio Inc. ARINC 664, Aircraft Data Network, Part 7: Avionics Full Duplex Switched Ethernet (AFDX) Network, 2005.

[4] Aeronautical Radio Inc. ARINC 429, ARINC specification 429-ALL: Mark 33 Digital Information Transfer System, 2001.

[5] Lauer, M. and Ermont, J. and Boniol, F. and Pagetti, C. : Latency and freshness analysis on IMA systems. 16th IEEE International Conference on Emerging Technologies and Factory Automation, Toulouse, France, September 2011

[6] Lauer, M. and Ermont, J. and Boniol, F. and Pagetti, C. : Analyzing end-to-end functional delays on an IMA platform. In Proc. of the 4th ISoLA'10, Heraklion, Greece, page 243-257. Springer-Verlag, 2010.

[7] N. Badache, K. Jaffres-Runser, J.-L. Scharbarg,C. Fraboul : End-to-end delay analysis in an Integrated Modular Avionics architecture. (short paper) ETFA 2013, Cagliari, Italy, Sep. 2013.

[8] M. Grenier, L. Havet, N. Navet : Configuring the communication on FlexRay- the case of the static segment. 4th European Congress on Embedded Real Time Software, Toulouse, France, January 2008.

[9] A. Al Sheikh and al. : Partitions scheduling on an IMA platform with strict periodicity and communication delays RTNS 2010, Toulouse, France.

[10] F. Martin and C. Fraboul : Modeling and Simulation of Integrated Modular Avionics Parallel and Distributed Processing. Proceedings of the Sixth Euromicro Workshop, Madrid, Spain, January 1998.

[11] Bauer, H. and Scharbarg, J. and Fraboul, C : Improving the Worst-Case Delay Analysis of an AFDX Network Using an Optimized Trajectory Approach IEEE Transactions on Industrial Informatics 2010, pages 521533.

[12] Charara, H. and Scharbarg, J. and Ermont, J. and Fraboul, C. : Methods for bounding end-to-end delays on an AFDX network 18th Euromicro Conference on Real-Time Systems, Dresden, Germany, July 2006. 